\title{
Intermediate-risk thyroid carcinoma: indicators of a poor prognosis
}

1 Unidade de Doenças da Tireoide, Divisão de Endocrinologia, Departamento de Medicina, Irmandade da Santa Casa de Misericórdia de São Paulo, São Paulo, SP, Brasil

${ }^{2}$ Faculdade de Ciências Médicas, Santa Casa de São Paulo, São Paulo, SP, Brasil

${ }^{3}$ Serviço de Medicina Nuclear, Irmandade da Santa Casa de Misericórdia de São Paulo, São Paulo, SP, Brasil
Correspondence to: Rosália do Prado Padovani Av. Ibirapuera 2.907, conj. 1.510 04029-000 - São Paulo, SP, Brasi rosaliapadovani@yahoo.com.br

Received on Jan/17/2020 Accepted on July/29/2020

DOI: 10.20945/2359-3997000000290
Fernanda Nascimento Faro

https://orcid.org/0000-0002-3240-075X

Ângela Maria Leal Barros Bezerra ${ }^{1}$

https://orcid.org/0000-0001-6760-857X

Nilza Maria Scalissi ${ }^{1,2}$

https://orcid.org/0000-0003-2158-6613

Adriano Namo Cury ${ }^{1,2}$

https://orcid.org/0000-0002-9777-8366

Marília Martins Marone ${ }^{3}$

https://orcid.org/0000-0002-5494-8281

Carolina Ferraz ${ }^{1,2}$

https://orcid.org/0000-0002-6620-8926

Rosália do Prado Padovani ${ }^{1,2,3}$

https://orcid.org/0000-0002-5497-6813

\begin{abstract}
Objective: The intermediate-risk (IR) category includes tumors with different degrees of aggression. We aimed to identify the risk factors associated with unfavorable response to initial treatment and compare the effect of low/high radioactive iodine (RAI) therapy. Subjects and methods: A total of 614 IR patients were selected from a database, during 1972-2015. All patients underwent total thyroidectomy and RAI therapy and were reclassified after 12-18 months into the favorable (complete/indeterminate) response group and the unfavorable (biochemical/incomplete structural) response group. A total of 92 patients were assessed for late response (mean: $9.19 \pm 5.73$ years). Age, gender, tumor size, histology, multifocality, vascular invasion, extrathyroidal extension, presence and number of lymph node metastasis, and stimulated thyroglobulin at ablation (sTg) were evaluated. Results: Mean age at diagnosis was $41.47 \pm 15.81$ years, and $83.6 \%$ of the patients were female. Within 12-18 months after initial therapy, unfavorable response was detected in $41.2 \%$ of the patients and was associated, in multivariate analysis, with lymph node metastasis ( $p=0.041$; odds ratio [OR] $=1.9)$, presence of more than five metastatic lymph nodes $(\mathrm{p}=0,017 ; \mathrm{OR}=2.6)$, and $\mathrm{sTg}>10 \mathrm{ng} /$ $\mathrm{mL}(\mathrm{p}=0.005 ; \mathrm{OR}=10.0)$. For patients with a longer follow-up, $\mathrm{sTg}>10 \mathrm{ng} / \mathrm{mL}$ was associated with unfavorable response $(p=0.002 ; O R=6.8)$. A higher $\mathrm{RAl}$ dose was not related to better prognosis at the end of the follow-up. Conclusion: $A$ sTg level of $>10 \mathrm{ng} / \mathrm{mL}$ and lymph node metastasis were associated with an unfavorable response 12-18 months after initial treatment. A RAI dose below 150 mCi was proven sufficient to treat IR patients. Arch Endocrinol Metab. 2020;64(6):764-71
\end{abstract}

\section{Keywords}

Thyroid neoplasms; thyroid cancer; differentiated thyroid cancer; radioactive iodine therapy; thyroglobulin; prognosis

\section{INTRODUCTION}

$\mathrm{T}$ he incidence of thyroid carcinoma, the most common endocrine cancer, has increased in recent decades $(1,2)$. Although the condition of most patients with differentiated thyroid cancer (DTC) improves when properly treated, a proportion of them (14\%-23\%) experience disease recurrence or do not respond to conventional therapies (3). To improve the effectiveness of the treatment, the management of DTC should be individualized. For this purpose, the 2015 American Thyroid Association (ATA) guidelines suggest that patients should be staged after surgery to assess the risk of recurrence and persistence of the disease in addition to the risk of mortality $(4,5)$.

Although initial staging is carried out, the recurrence and persistence rates within the same recurrence and persistence risk category may vary. For intermediate risk (IR), which is the second most common category of DTC (25\%-35\% of cases) (6-8), variations in the recurrence and persistence rates are relatively large (3\%$30 \%$ ) (5). This category includes a very heterogeneous population group. Within the same risk classification, 
it is possible to identify malignant tumors with a combination of different risk characteristics and, consequently, different degrees of aggressiveness.

Therefore, it remains unclear whether all patients classified in the IR category should receive the same initial treatment, especially radioactive iodine therapy (RAI) as adjunct therapy and perform the prescribed activity $(5,6,9-11)$.

Considering the importance of differentiating patients with "low-risk characteristics" from those with "high-risk characteristics," this study aimed to identify risk factors associated with tumor persistence/recurrence disease in a cohort of patients with intermediate risk of DTC recurrence. We also aimed to assess whether the use of a lower or a higher RAI dose $(\leq 150$ and $>150$ $\mathrm{mCi}$ ) influences the outcome of these patients.

\section{SUBJECTS AND METHODS}

\section{Patients and study design}

A total of 614 IR patients were selected from the database of DTC of the Nuclear-Medicine Service, Nuclimagem, from 1972 to 2015. Nuclimagem is a nuclear medicine service that provides specialized care to patients in Santa Casa de Misericórdia de São Paulo. It receives patients not only from Santa Casa but also from all regions of the state, referred by the São Paulo State Health secretary to undergo treatments or examinations. That is why a long follow-up is not available for most of these patients.

All patients selected from the database fulfilled the ATA criteria for determine the IR of recurrence and had at least one of the following characteristics: i) aggressive histology (tall cell, columnar, insular, and solid variant), ii) T3 with microscopic invasion of the tumor into the perithyroidal soft tissues at initial surgery, iii) papillary thyroid cancer with vascular invasion, or iv) T-Nl (more than five lymph nodes involved whose sizes were between 0.2 and $3.0 \mathrm{~cm}$ without extranodal extension). Patients with positive anti-thyroglobulin antibodies ( $\mathrm{TgAb}$ ) were excluded from the study.

All patients underwent total thyroidectomy and RAI therapy with doses between 100 and $300 \mathrm{mCi}$ after thyroid hormone withdrawal and were placed on a lowiodine diet in line with the ATA recommendations. A RAI dose of up to $150 \mathrm{mCi}$ was considered as a low dose, while a dose above $150 \mathrm{mCi}$ was considered as a high dose. After initial therapy, levothyroxine suppressive therapy was administered, as recommended by the ATA guidelines (5).

\section{Outcomes}

Twelve to eighteen months after initial treatment (first assessment after ablation), all patients were reevaluated by measuring the levels of thyroid stimulating hormone (TSH)-stimulated thyroglobulin ( $\mathrm{Tg}$ ), and by performing a neck ultrasound (US), with or without a diagnostic whole-body scintigraphy (WBS). Clinical status was defined by classifying patients' response to initial treatment as excellent, indeterminate, biochemical incomplete, and structural incomplete, according to the 2015 ATA classification (5). Patients with excellent and indeterminate responses were classified as having a favorable response. On the contrary, biochemical or structural incomplete responses were classified as an unfavorable response.

Excellent response was considered if the imaging findings were negative and the patient had either a suppressed Tg level of $<0.2 \mathrm{ng} / \mathrm{mL}$ or a stimulated $\mathrm{Tg}$ (sTg) level of $<1.0 \mathrm{ng} / \mathrm{mL}$. Indeterminate response indicated nonspecific findings on imaging studies, faint uptake in the thyroid bed on RAI scanning, and a nonstimulated $\mathrm{Tg}$ detectable level of $\geq 0.2$ but $<1.0$ $\mathrm{ng} / \mathrm{mL}$ or a $\mathrm{sTg}$ detectable level $\geq 1.0$ but $<10 \mathrm{ng} /$ $\mathrm{mL}$. Biochemical incomplete response was reported if the imaging findings were negative and the patient had either a suppressed Tg level of $\geq 1.0 \mathrm{ng} / \mathrm{mL}$ or a $\mathrm{sTg}$ level of $\geq 10 \mathrm{ng} / \mathrm{mL}$. Structural incomplete response was indicated if there was presence of structural or functional evidence of disease with any $\mathrm{Tg}$ level.

Of the total patients selected from the database of the nuclear medicine service, 92 were examined in our endocrinology outpatient clinic (Irmandade da Santa Casa de Misericórdia de São Paulo). Therefore institution's, we were able to perform a long-term follow-up of these patients (mean: $9.19 \pm 5.73$ years) and reevaluate their response to the proposed treatment at the end of the follow-up.

\section{Study variables}

The following risk factors were evaluated: age at diagnosis $(<55$ and $\geq 55$ years $)$, gender, tumor size ( $\leq 1 \mathrm{~cm}, 1-4 \mathrm{~cm}$, and $\geq 4 \mathrm{~cm}$ at the largest diameter), histology (papillary or follicular carcinoma), histology of papillary carcinoma (classic, follicular, and others), histology of follicular carcinoma (minimally invasive or extensive invasive), multifocality, vascular invasion, extrathyroidal extension, presence and number of lymph node metastasis ( $\leq 5$ and $>5$ metastatic lymph nodes), RAI doses $(\leq 150 \mathrm{mCi}$ and $>150 \mathrm{mCi})$, and $\mathrm{sTg}$ at ablation ( $<5 \mathrm{ng} / \mathrm{mL}, 5-10 \mathrm{ng} / \mathrm{mL}$, and $>10 \mathrm{ng} / \mathrm{mL})$. 


\section{Assays}

All patients included in our study underwent the first RAI treatment until 2015.

The analyses of sTg at ablation and $\mathrm{TgAb}$ were performed in the same laboratory and the levels were assessed using the immulite thyroglobulin assay. This is a first-generation $\mathrm{Tg}$ assay with a functional sensitivity (FS) of $1.0 \mathrm{ng} / \mathrm{ml}$ and a lower limit of detection of 0.2 $\mathrm{ng} / \mathrm{mL}$.

For patients who were assessed for response to initial treatment until 2015, sTg was used because, until then, second-generation $\mathrm{Tg}$ assays were not available in our laboratory.

After 2015, patients' response to initial treatment was evaluated by measuring the level of suppressed or sTg because, at that time, second-generation $\mathrm{Tg}$ assays started to become available in our institution. Hence, thereafter, Tg levels were assessed using the chemiluminescent assay (Access Thyroglobulin Assay; Beckman Coulter) with an FS of $0.1 \mathrm{ng} / \mathrm{mL}$.

\section{Imaging methods}

Neck US was performed by experienced ultrasonographers from our institution using a highresolution color Doppler US apparatus with a 7.5$\mathrm{MHz}$ linear transducer. Diagnostic or post-therapeutic 131I-WBS was performed using a one-head - camera (Apex SPX 4000; Elscint Italia, Milan, Italy) with a high-energy collimator and with a sensitivity of 160 $\mathrm{cpm} / \mathrm{mCi}$. The scan speed was $10 \mathrm{~cm} / \mathrm{min}$ with total counts of at least $100,000 \mathrm{cpm}$.

\section{Statistical analysis}

Statistical analysis was performed using SPSS version 20.0. Categorical variables were expressed as absolute and relative frequencies and were compared using chi-square or Fisher's exact test when appropriate. Continuous variables were expressed as mean and standard deviation.

Univariate analysis and multivariate logistic regression models were used to investigate the association between unfavorable response and clinical variables. A multivariate logistic regression model was used to identify independent prognostic factors of recurrence. Odds ratios (ORs) were calculated for the variables that were significant in the univariate and multivariate analyses. A p value of $<0.05$ was considered to indicate statistical significance.
To evaluate the effect of RAI activities on prognosis, we analyzed the association between RAI doses $(\leq 150$ and $>150 \mathrm{mCi}$ ) and an unfavorable response at the end of the follow-up. This association was exclusively evaluated in the subgroup of patients with an initial unfavorable response, considering the hypothesis that this particular group would more likely benefit from higher doses of RAI.

The study was approved by the local research ethics committee.

\section{RESULTS}

A total of 614 patients diagnosed with intermediaterisk DTC according to the 2015 ATA risk stratification guidelines were studied. Mean age at diagnosis was $41.47 \pm 15.81$ years. Approximately $83.6 \%$ of the total patients were female, and $86.8 \%$ had papillary thyroid carcinoma. Moreover, $44.8 \%$ of the patients had lymph node metastasis. The serum $\mathrm{Tg}$ value at ablation was less than $5 \mathrm{ng} / \mathrm{mL}$ in $87.9 \%$ of the patients, and $61 \%$ received high RAI therapy $(>150 \mathrm{mCi})$. When patients were reevaluated 12-18 months after the initial therapy, $58.8 \%$ achieved a favorable response (complete or indeterminate), while $41.2 \%$ showed unfavorable response (biochemical or structural incomplete) (Table 1). The frequency of incomplete structural response was higher in males, when compared to incomplete biochemical response $(\mathrm{p}=0.031 ; \mathrm{OR}=$ 2.08 [95\% confidence interval (CI): 1.06-4.04]).

In the univariate analysis, the risk factors associated with unfavorable response 12-18 months after initial therapy were male gender $(\mathrm{p}=0.022)$, tumor size $>4$ $\mathrm{cm}(\mathrm{p}=0.038)$, presence of lymph node metastasis $(\mathrm{p}<0.001)$, more than five lymph node metastasis $(\mathrm{p}<0.001)$, and serum sTg value at ablation higher than $10 \mathrm{ng} / \mathrm{mL}(\mathrm{p}<0.001)$ (Table 2). Multivariate analysis confirmed the association between unfavorable response and lymph node metastasis, number of lymph node metastasis, and sTg value at ablation (Table 3 ).

Of the 92 patients who were evaluated after a longterm follow-up (mean: $9.19 \pm 5.73$ years), $65.2 \%$ showed a favorable response 12-18 months after initial therapy (Table 4). The majority of the patients maintained a favorable response, and only $3.3 \%$ had favorable to unfavorable response. Of the patients initially classified as having an unfavorable response, $31.3 \%$ showed a favorable response at the end of the follow-up (Table 4). 
Table 1. Baseline characteristics of 614 intermediate-risk DTC patients according to the American Thyroid Association risk stratification

\begin{tabular}{|c|c|c|}
\hline Characteristics & $\%$ & $\mathbf{n}$ \\
\hline \multicolumn{3}{|l|}{ Age (years) at diagnosis } \\
\hline$<55$ & $77.2 \%$ & 474 \\
\hline$\geq 55$ & $22.8 \%$ & 140 \\
\hline \multicolumn{3}{|l|}{ Gender } \\
\hline Female & $83.6 \%$ & 513 \\
\hline Male & $16.4 \%$ & 101 \\
\hline \multicolumn{3}{|l|}{ Histology } \\
\hline Papillary & $86.8 \%$ & 533 \\
\hline Follicular & $13.2 \%$ & 81 \\
\hline \multicolumn{3}{|l|}{ Histology variant of papillary carcinoma } \\
\hline Classic & $65.1 \%$ & 343 \\
\hline Folicular & $27.9 \%$ & 147 \\
\hline Others & $7.0 \%$ & 37 \\
\hline \multicolumn{3}{|l|}{ Histology variant of follicular carcinoma } \\
\hline Minimally invasive & $93.5 \%$ & 72 \\
\hline Extensively invasive & $6.5 \%$ & 5 \\
\hline \multicolumn{3}{|l|}{ Tumor size (cm) } \\
\hline$\leq 1$ & $18.3 \%$ & 97 \\
\hline$>1-4$ & $53.1 \%$ & 281 \\
\hline$\geq 4$ & $28.5 \%$ & 151 \\
\hline Multifocality & $30.9 \%$ & 190 \\
\hline Extrathyroidal invasion & $17.9 \%$ & 110 \\
\hline Vascular invasion & $37.4 \%$ & 201 \\
\hline Presence of metastatic lymph nodes & $44.8 \%$ & 275 \\
\hline \multicolumn{3}{|l|}{ Number of metastatic lymph nodes } \\
\hline$\leq 5$ & $81.2 \%$ & 437 \\
\hline$>5$ & $18.8 \%$ & 101 \\
\hline \multicolumn{3}{|l|}{ Stimulated $\mathrm{Tg}$ at ablation (ng/mL) } \\
\hline$<5$ & $87.9 \%$ & 333 \\
\hline $5-10$ & $3.7 \%$ & 14 \\
\hline$>10$ & $8.4 \%$ & 32 \\
\hline \multicolumn{3}{|l|}{ RAl dose (mCi) } \\
\hline$\leq 150$ & $39.0 \%$ & 225 \\
\hline$>150$ & $61.0 \%$ & 352 \\
\hline \multicolumn{3}{|l|}{ Response classification to initial therapy } \\
\hline Complete & $29.2 \%$ & 179 \\
\hline Indeterminate & $29.6 \%$ & 182 \\
\hline Biochemical incomplete & $27.2 \%$ & 167 \\
\hline Structural incomplete & $14.0 \%$ & 86 \\
\hline
\end{tabular}

A total of $14.1 \%$ of the patients developed distant metastasis, $16.4 \%$ underwent another surgery, and $19.5 \%$ received another dose of RAI. We also observed that patients who achieved an excellent response to therapy in the first 2 years of evaluation had a substantially lower risk of recurrence/persistence than those who presented an incomplete response to therapy. Moreover, 93\% of patients who had a complete response in the initial stratification had the same response at the end of the follow-up. Regarding patients who had an incomplete response at the initial assessment, $58 \%$ achieved the same response in the final evaluation (Table 5 ).

The characteristic associated with an unfavorable response at the end of the follow-up in the univariate $(\mathrm{p}$ $=0,002)$ and multivariate $(\mathrm{p}=0.002 ; \mathrm{OR}=6.87[95 \%$ CI: 2.03-23.2]) analyses was sTg at ablation higher than $10 \mathrm{ng} / \mathrm{mL}$ (Table 6).

With regard to the effect of high and low RAI doses on patients' prognosis, no significant association was observed between RAI doses and response range at the end of the follow-up ( $\mathrm{p}=0.699)$, demonstrating that using RAI doses higher than $150 \mathrm{mCi}$ had no effect on long-term outcomes (Table 7).

\section{DISCUSSION}

The IR category proposed by the 2015 ATA guideline is very heterogeneous and comprises tumors of different degrees of aggressiveness. Low-risk and highrisk patients should be provided with individualized treatment and should not receive insufficient or unnecessary therapies. In addition, recognizing highrisk patients is of utmost importance to recommend a more aggressive approach, and these patients should be closely followed up to determine who among them will benefit from the treatment.

In this sense, the 2015 ATA guideline recommends the selective use of RAI for IR patients. In fact, RAI treatment should be considered for patients of this category but who also have a higher risk of persistent or recurrent disease. However, which characteristics represent an important risk of recurrence or persistence and who among the IR patients deserve a more aggressive treatment remain controversial (5). So, the main objective of this study was to identify risk factors associated with tumor persistence/recurrence disease in a cohort of patients with intermediate risk of DTC recurrence.

First, it is important to highlight some of the characteristics of this study. The present study used a large sample size and determined the patients' response 
Table 2. Univariate analysis of factors associated with incomplete response 12-18 months after initial therapy and the OR of significant factors

\begin{tabular}{|c|c|c|c|c|c|c|c|c|}
\hline \multirow{2}{*}{ Characteristics } & & \multirow{2}{*}{$\begin{array}{c}\text { Complete/ } \\
\text { Indeterminate }\end{array}$} & \multirow{2}{*}{ Incomplete } & \multirow{2}{*}{$P$ value } & \multirow{2}{*}{ Test } & \multirow{2}{*}{$\mathbf{O} \mathbf{R}^{\star}$} & \multicolumn{2}{|c|}{$95 \% \mathrm{Cl}$ for $\mathrm{OR}$} \\
\hline & & & & & & & Lower & Upper \\
\hline \multirow[t]{2}{*}{ Age (years) at diagnosis } & $<55$ & 282 & 192 & 0.517 & Chi-square & & & \\
\hline & $\geq 55$ & 79 & 61 & & & & & \\
\hline \multirow[t]{2}{*}{ Gender } & Female & 312 & 201 & 0.022 & Chi-square & $1^{*}$ & & \\
\hline & Male & 49 & 52 & & & 1.65 & 1.07 & 2.53 \\
\hline \multirow[t]{2}{*}{ Histology } & Papillary & 307 & 226 & 0.122 & Chi-square & & & \\
\hline & Follicular & 54 & 27 & & & & & \\
\hline \multirow[t]{3}{*}{ Histology variant of papillary carcinoma } & Classic & 191 & 152 & 0.312 & Chi-square & & & \\
\hline & Folicular & 88 & 59 & & & & & \\
\hline & Others & 25 & 12 & & & & & \\
\hline \multirow[t]{2}{*}{ Histology variant of follicular carcinoma } & Minimally invasive & 49 & 23 & 0.329 & Fisher's Exact & & & \\
\hline & Extensively invasive & 2 & 3 & & & & & \\
\hline \multirow[t]{3}{*}{ Tumor size (cm) } & $\leq 1$ & 69 & 28 & 0.038 & Chi-square & $1^{*}$ & & \\
\hline & $>1-4$ & 169 & 112 & & & 1.63 & 0.99 & 2.69 \\
\hline & $\geq 4$ & 83 & 68 & & & 2.02 & 1.17 & 3.48 \\
\hline \multirow[t]{2}{*}{ Multifocality } & No & 253 & 171 & 0.511 & Chi-square & & & \\
\hline & Yes & 108 & 82 & & & & & \\
\hline \multirow[t]{2}{*}{ Extrathyroidal invasion } & No & 293 & 211 & 0.477 & Chi-square & & & \\
\hline & Yes & 68 & 42 & & & & & \\
\hline \multirow[t]{2}{*}{ Vascular invasion } & No & 187 & 150 & 0.128 & Chi-square & & & \\
\hline & Yes & 125 & 76 & & & & & \\
\hline \multirow[t]{2}{*}{ Presence of metastatic lymph nodes } & No & 227 & 112 & 0.000 & Chi-square & $1^{*}$ & & \\
\hline & Yes & 134 & 141 & & & 2.13 & 1.54 & 2.96 \\
\hline \multirow[t]{2}{*}{ Number of metastatic lymph nodes } & $\leq 5$ & 272 & 165 & 0.000 & Chi-square & $1^{*}$ & & \\
\hline & $>5$ & 40 & 61 & & & 2.51 & 1.61 & 3.92 \\
\hline \multirow[t]{3}{*}{ Stimulated $\mathrm{Tg}$ at ablation (ng/mL) } & $<5$ & 214 & 119 & 0.000 & Chi-square & $1^{*}$ & & \\
\hline & $5-10$ & 13 & 1 & & & 0.14 & 0.02 & 1.07 \\
\hline & $>10$ & 7 & 25 & & & 6.42 & 2.70 & 15.29 \\
\hline
\end{tabular}

* OR reference: category 1; OR: odds ratio; Cl: confidence interval; Tg: serum thyroglobulin; RAl: radioactive iodine.

Significant difference at $p \leq 0.05$.

Table 3. Factors associated with incomplete response by multivariate analysis

\begin{tabular}{|c|c|c|c|c|c|}
\hline \multirow{2}{*}{ Characteristics } & & \multirow{2}{*}{ OR } & \multicolumn{2}{|c|}{$95 \% \mathrm{Cl}$ for $\mathrm{OR}$} & \multirow{2}{*}{$P$ value } \\
\hline & & & Lower & Upper & \\
\hline Presence of metastatic lymph nodes & Yes & 1.905 & 1.028 & 3.528 & 0.041 \\
\hline Number of metastatic lymph nodes & $>5$ & 2.634 & 1.187 & 5.846 & 0.017 \\
\hline Stimulated $\mathrm{Tg}$ at ablation $(\mathrm{ng} / \mathrm{mL})$ & $>10$ & 10.095 & 2.044 & 49.863 & 0.005 \\
\hline
\end{tabular}

* OR: odds ratio; Cl: confidence interval; Tg: serum thyroglobulin; RAl: radioactive iodine.

Significant difference at $p \leq 0.05$.

All factors that were significant in the univariate analysis were included in the multivariate analysis (Table 2): gender, tumor size, stimulated Tg at ablation, and presence and number of metastatic lymph nodes.

Table 4. Evaluation of 92 intermediate-risk DTC patients according to response range 12-18 months after initial therapy and after a long-term follow-up (mean; $9.19 \pm 5.73$ years)

\begin{tabular}{llcc} 
& & \multicolumn{2}{c}{ Response range at the end of follow-up } \\
\cline { 3 - 3 } & Response range after 12-18 months of initial therapy & Unfavorable \\
\cline { 2 - 3 } & Favorable & 58 & 2 \\
\hline
\end{tabular}

* Favorable: complete or indeterminate response. Unfavorable: biochemical/structural incomplete response.

Significant difference at $p \leq 0.05$. 
Table 5. Initial and final response of 92 patients who had a longer follow-up

\begin{tabular}{|c|c|c|c|c|c|}
\hline \multirow{2}{*}{ Initial Response Type } & \multicolumn{4}{|c|}{ Final Response Type } & \multirow{2}{*}{ Tota } \\
\hline & Complete & Indeterminate & Biochemical incomplete & Structural incomplete & \\
\hline Complete & $93 \%$ & $3 \%$ & $0 \%$ & $5 \%$ & $100 \%$ \\
\hline Indeterminate & $60 \%$ & $40 \%$ & $0 \%$ & $0 \%$ & $100 \%$ \\
\hline Biochemical incomplete & $23 \%$ & $8 \%$ & $54 \%$ & $15 \%$ & $100 \%$ \\
\hline Structural incomplete & $21 \%$ & $11 \%$ & $11 \%$ & $58 \%$ & $100 \%$ \\
\hline Total & $61 \%$ & $13 \%$ & $10 \%$ & $16 \%$ & $100 \%$ \\
\hline
\end{tabular}

Table 6. Factors associated with incomplete response after a long-term follow-up (mean: $9.19 \pm 5.73$ years)

\begin{tabular}{|c|c|c|c|c|c|c|c|c|c|c|c|}
\hline \multirow{3}{*}{ Characteristics } & & \multirow{3}{*}{ Favorable } & \multirow{3}{*}{ Unfavorable } & \multirow{3}{*}{$P$ value } & \multicolumn{3}{|c|}{ Univariate analysis } & \multirow{3}{*}{$P$ value } & \multicolumn{3}{|c|}{ Multivariate analysis } \\
\hline & & & & & \multirow{2}{*}{ OR } & \multicolumn{2}{|c|}{ 95\% Cl for OR } & & \multirow{2}{*}{$\mathrm{OR}$} & \multicolumn{2}{|c|}{$95 \% \mathrm{Cl}$ for OR } \\
\hline & & & & & & Lower & Upper & & & Lower & Upper \\
\hline \multirow{3}{*}{$\begin{array}{l}\text { sTg } \\
\text { at ablation (ng/mL) }\end{array}$} & $<5$ & 38 & 7 & 0.002 & $1^{*}$ & & & & & & \\
\hline & $5-10$ & 6 & 2 & & 1.81 & 0.30 & 10.86 & & & & \\
\hline & $>10$ & 8 & 12 & & 8.14 & 2.44 & 27.15 & 0.002 & 6.875 & 2.030 & 23.283 \\
\hline Constant & & & & & & & & 0,000 & 0,200 & & \\
\hline
\end{tabular}

* OR reference: category 1; OR: odds ratio; Cl: confidence interval; sTg: stimulated serum thyroglobulin. Significant difference at $p \leq 0.05$.

Table 7. RAl doses and response range at the end of the follow-up

\begin{tabular}{lcccc}
\hline Characteristics & & Favorable & Unfavorable & P value \\
\hline RAl dose $(\mathrm{mCl})$ & $\leq 150$ & 5 & 8 & 0,699 \\
& $>150$ & 5 & 14 & \\
\hline
\end{tabular}

* RAl: radioactive iodine.

Significant difference at $p \leq 0.05$.

to initial therapy at the first 12-18 months after RAI; we also evaluated the long-term (mean: $9.19 \pm 5.73$ years) results of a significant number of patients. Furthermore, all patients initially underwent the same surgical treatment and received RAI treatment in the same institution using the same protocol.

Our study demonstrated, using a large cohort of IR patients at risk for recurrence and persistent disease, that the risk factors associated with unfavorable response to initial therapy were male gender, larger tumor size $(>4 \mathrm{~cm})$, presence of lymph node metastasis, and a $\mathrm{sTg}$ value at ablation higher than $10 \mathrm{ng} / \mathrm{mL}$. Lymph node metastasis, number of metastatic lymph nodes, and level of sTg at ablation were considered independent risk factors for incomplete response 12-18 months after the initial treatment; moreover, sTg was associated with incomplete response in a longer follow-up.

Bandeira and cols. (12), Bernier and cols. (13), and Ballal and cols. (7) showed the prognostic value of sTg measured before RAI therapy and suggested that a higher sTg value at this point, the greater the likelihood of an incomplete response to initial treatment. Bandeira and cols. (12) proposed that $3.75 \mathrm{ng} / \mathrm{mL}$ is a good cut-off value for incomplete response. Meanwhile, Ballal and cols. (7) demonstrated that a sTg level at first follow-up higher than $10 \mathrm{ng} / \mathrm{mL}$ was a risk factor for a significant reduction in event-free survival. Our findings corroborate with the results of these studies and emphasized the importance of checking the sTg value after surgery to help clinicians decide the appropriate RAI therapy. Inappropriately elevated postoperative $\mathrm{Tg}$ values are being used as a basis to recommend the appropriate RAI adjuvant therapy, even in the absence of structural disease $(7,12-18)$.

The ATA guideline also recommends RAI adjuvant therapy in older patients with large primary tumors ( $>45$ years, $>4 \mathrm{~cm}$ ) because of the significant risk of recurrence or distant metastasis (5). Tuttle and Sabra and Ballal and cols. $(7,9)$ demonstrated that these variables were associated with an incomplete response 
and that patients with these characteristics can benefit from adjuvant treatment with RAI (100-150 mCi). Our results were in line with their data regarding tumor size bigger than $4 \mathrm{~cm}$ but not regarding the association of age worst prognosis.

The relationship between DTC recurrences and gender was evaluated in some studies. Only one previous study showed less disease recurrence in female patients, with another study noting less recurrence in this group after conducting only univariate analysis. Although most of these studies showed some positive effect of female gender on overall survival and diseasespecific survival (DSS), a female survival advantage is not a universal finding, and the effect of gender on recurrences of DTC was not substantiated (19). In our study, male gender was a risk factor for an unfavorable response; however, when adjusted for other variables, it was not significant, showing that the influence of gender on prognosis depends on other characteristics.

Compared with that of incomplete biochemical response, the frequency of incomplete structural response was more prevalent in males than in females. This results corroborate with Nilubol and cols.'s findings (20), which showed that men with thyroid cancer are more likely to have more advanced and aggressive cancer, although this study did not conclude that male gender was an independent predictor of DSS.

In accordance with the findings reported in literature, vascular invasion increases the risk of distant metastases. Hence, RAI therapy should be considered in these patients to facilitate initial staging and followup (21). In the present study, vascular invasion was not associated with an unfavorable response, which is inconsistent with the findings of previous publication.

Regarding lymph node involvement, the current guidelines do not recommend the use of RAI therapy for all patients with lymph node metastases $(3,5)$. Tuttle and Sabra (9) and Ballal and cols. (7) proposed the selective use of RAI only in high-risk patients with $\mathrm{Nl}$ disease (more than five involved lymph nodes), considering that these patients carry a risk of recurrence $(>20 \%)$. Consistent with these findings, our study also demonstrated that the presence of lymph node involvement in IR patients should be considered as an independent risk factor for incomplete response $(5,7)$, especially in the presence of more than five metastatic lymph nodes, and an indication for adjuvant RAI therapy.

A differential of this study was the comparison between the effect of different RAI doses in the IR patients' response to the initial treatment. To the best of our knowledge, only a few studies in literature compared the effect of different RAI doses in IR patients with different risk characteristics. Castagna and cols. (6) was one of the authors who carried out a study to compare the effect of RAI doses below and above $100 \mathrm{mCi}(3,700 \mathrm{MBq})$ in the success of the ablation and in the outcome of IR patients. Similar to our results, no significant difference was observed in terms of the effect between these activities. Our data also showed no benefit related to the response to initial treatment in patients treated with high dose of RAI $(>150 \mathrm{mCi})$. We analyzed the response after 12-18 months and after a mean of 9 years. In the subgroup initially classified as having an incomplete response, no significant difference was observed between lower or higher doses of RAI.

Moreover, our study reported that $96.7 \%$ of the patients initially classified as having a favorable response and $58 \%$ patients initially classified as having an incomplete response maintained this status after 9 years of follow-up. These findings are consistent with the results reported in literature (22).

Unfortunately, a long-term follow-up of these patients was not performed, and we consider this as a limitation of the study. However, the assessment of 92 patients who have been followed up for a longer period confirmed that a risk-adapted approach to follow-up cannot be based solely on static, initial estimates of risk, as previously suggested by other authors $(5,11)$. Vaisman and cols. clearly showed the importance of the restratification in several cohorts worldwide and in Brazil (22).

The other limitations of the present study are follows: although RAI was administered in the same laboratory, using the same protocols and type of preparation, surgery and follow-up of patients were not carried out at the same center. The Nuclear Medicine Division of Santa Casa de São Paulo, as a referral center, receives patients from several other centers in the region to undergo RAI therapy. Consequently, variables such as surgical ability, extent of surgery (with or without lymphadenectomy), and follow-up protocols specific to each service may be considered as confounding factors $(6,12)$.

In conclusion, our study provides further evidence that some risk factors are associated with worst prognosis in IR DTC patients, specifically the presence of lymph node metastasis, number of involved nodes, and high serum $\mathrm{Tg}$ levels at ablation, which increase 
the risk of an unfavorable response. For IR patients, sTg ablation should be evaluated and values above 10 $\mathrm{ng} / \mathrm{mL}$ should be considered an indication for a more aggressive treatment and a closer follow-up. In the context of adjuvant therapy, doses lower or equal to $150 \mathrm{mCi}$ appear to be sufficient for treating IR patients.

Funding: this research did not receive any specific grant from any funding agency in the public, commercial, or not-for-profit sector.

Acknowledgments: we would like to thank Editage (www. editage.com) for English language editing.

Disclosure: no potential conflict of interest relevant to this article was reported.

\section{REFERENCES}

1. Howlader N, Noone AM, Krapcho M, Miller D, Brest A, Yu M, et al. SEER Cancer Statistics Review, 1975-2016. National Cancer Institute. Available from: https://seer.cancer.gov/csr/1975_2016/.

2. Davies L, Welch HG. Current thyroid cancer trends in the United States. JAMA Otolaryngol Head Neck Surg. 2014;140(4):317-22.

3. Rosário PW, Ward LS, Carvalho GA, Graf H, Maciel RMB, Maciel LMZ, et al. Nódulo tireoidiano e câncer diferenciado de tireoide: atualização do consenso brasileiro. Arq Bras Endocrinol Metab. 2013;57(4):240-64.

4. Tuttle M, Haugen B, Perrier ND. Updated American Joint Committee on Cancer/Tumor-Node-Metastasis Staging System for Differentiated and Anaplastic Thyroid Cancer (Eighth Edition): What Changed and Why? Thyroid. 2017;27(6):751-6.

5. Haugen BR, Alexander EK, Bible KC, Doherty GM, Mandel SJ, Nikiforov YE, et al. American Thyroid Association Management Guidelines for Adult Patients with Thyroid Nodules and Differentiated Thyroid Cancer: The American Thyroid Association Guidelines Task Force on Thyroid Nodules and Differentiated Thyroid Cancer. Thyroid. 2016;26(1):1-133.

6. Castagna MG, Cevenini G, Theodoropoulou A, Maino F, Memmo S, Claudia C, et al. Post-surgical thyroid ablation with low or high radioiodine activities results in similar outcomes in intermediate risk differentiated thyroid cancer patients. Eur J Endocrinol. 2013;169(1):23-9.

7. Ballal S, Soundararajan R, Garg A, Chopra S, Bal C. Intermediaterisk differentiated thyroid carcinoma patients who were surgically ablated do not need adjuvant radioiodine therapy: long-term outcome study. Clin Endocrinol (Oxf). 2016;84(3):408-16.

8. Triviño Ibáñez EM, Muros MA, Torres Vela E, Llamas Elvira JM. The role of early 18F-FDG PET/CT in therapeutic management and ongoing risk stratification of high/intermediate-risk thyroid carcinoma. Endocrine. 2016;51(3):490-8.

9. Tuttle RM, Sabra MM. Selective use of RAl for ablation and adjuvant therapy after total thyroidectomy for differentiated thyroid cancer: a practical approach to clinical decision making. Oral Oncol. 2013;49(7):676-83.

10. Ha S, Oh SW, Kim YK, Koo do H, Jung YH, Yi KH, et al. Clinical Outcome of RemnantThyroid Ablation with Low Dose Radioiodine in Korean Patients with Low to Intermediate-risk Thyroid Cancer. J Korean Med Sci. 2015;30(7):876-81.

11. Tuttle RM, Tala H, Shah J, Leboeuf R, Ghossein R, Gonen M, et al. Estimating risk of recurrence in differentiated thyroid cancer after total thyroidectomy and radioactive iodine remnant ablation: using response to therapy variables to modify the initial risk estimates predicted by the new American Thyroid Association staging system. Thyroid. 2010;20(12):1341-9.

12. Bandeira L, Padovani RDP, Ticly AL, Cury AN, Scalissi NM, Marone MMS, et al. Thyroglobulin levels before radioactive iodine therapy and dynamic risk stratification after 1 year in patients with differentiated thyroid cancer. Arch Endocrinol Metab. 2017;61(6):590-9.

13. Bernier MO, Morel O, Rodien P, Muratet JP, Giraud P, Rohmer V, et al. Prognostic value of an increase in the serum thyroglobulin level at the time of the first ablative radioiodine treatment in patients with differentiated thyroid cancer. Eur J Nucl Med Mol Imaging. 2005;32(12):1418-21.

14. de Rosário PW, Guimarães VC, Maia FF, Fagundes TA, Purisch S, Padrao EL, et al. Thyroglobulin before ablation and correlation with posttreatment scanning. Laryngoscope. 2005;115(2):264-7.

15. Kim TY, Kim WB, Kim ES, Ryu JS, Yeo JS, Kim SC, et al. Serum thyroglobulin levels at the time of 131 remnant ablation just after thyroidectomy are useful for early prediction of clinical recurrence in low-risk patients with differentiated thyroid carcinoma. J Clin Endocrinol Metab. 2005;90(3):1440-5.

16. Heemstra KA, Liu YY, Stokkel M, Kievit J, Corssmit E, Pereira AM, Romijn JA, et al. Serum thyroglobulin concentrations predict disease-free remission and death in differentiated thyroid carcinoma. Clin Endocrinol (Oxf). 2007;66(1):58-64.

17. Ronga G, Filesi M, Ventroni G, Vestri AR, Signore A. Value of the first serum thyroglobulin level after total thyroidectomy for the diagnosis of metastases from differentiated thyroid carcinoma. Eur J Nucl Med. 1999;26(11):1448-52.

18. Melo M, Costa G, Ribeiro C, Carrilho F, Martins MJ, da Rocha $A G$, et al. Stimulated thyroglobulin at recombinant human TSHaided ablation predicts disease-free status one year later. J Clin Endocrinol Metab. 2013;98(11):4364-72.

19. Jonklaas J, Nogueras-Gonzalez G, Munsell M, Litofsky D, Ain KB, Bigos ST, et al. The impact of age and gender on papillary thyroid cancer survival. J Clin Endocrinol Metab. 2012;97(6):E878-87.

20. Nilubol N, Zhang L, Kebebew E. Multivariate analysis of the relationship between male sex, disease-specific survival, and features of tumor aggressiveness in thyroid cancer of follicular cell origin. Thyroid. 2013;23(6):695-702.

21. Vaisman F, Tala H, Grewal R, Tuttle RM. In differentiated thyroid cancer, an incomplete structural response to therapy is associated with significantly worse clinical outcomes than only an incomplete thyroglobulin response. Thyroid. 2011;21 (12):1317-22.

22. Vaisman F, Momesso D, Bulzico DA, Pessoa CH, Dias F, Corbo $\mathrm{R}$, et al. Spontaneous remission in thyroid cancer patients after biochemical incomplete response to initial therapy. Clin Endocrinol (Oxf). 2012;77(1):132-8. 